\title{
Determination of Additional Aperture in Non-Metal Sewer Pipes by Image Processing
}

\author{
Mohamed Qays Jameel Alsalihi \\ Department of Computer Engineering \\ Selcuk University
}

Turkey, 2017

\author{
Murat Selek, PhD \\ Department of computer engineering \\ Selcuk University \\ Turkey, 2017
}

\begin{abstract}
In this investigation, image processing was applied to find out the disorder may accrue in the non-metal sewer underground pipelines (i.e. additional aperture). This disorder may be discovered via extracting the characteristics from interior image taken and tackled through suitable filters to improve its appearance, and ultimately subjected to feature extraction process to extract and determine their characters, and at last step, finding out and diagnosing the faults and disadvantages that may exist in the tested pipelines, automatically, without the need to the human eye. For detecting the additional apertures, edge detection technique was used. By this technique a reliable result was achieved. An image with additional apertures was produced.
\end{abstract}

\section{General Terms}

Image processing techniques

\section{Keywords}

Edge detection technique, Sobel, and Prewitt operators

\section{INTRODUCTION}

Image processing was applied in the mid-1960s when the third generation computers provided rapid and wide storage capacity for using algorithmic techniques in image processing implementation. Since then, this field has witnessed outstanding activities and development. Therefore, image processing attracted the investigators in this fields for more research and investigation. Image processing also was used in many other fields of knowledge, such as statistics, computer science, information, physics, chemistry engineering, biology, and medicine [1].

Image Processing (IP) is a mechanism to improve raw images delivered by photographing devices located on satellites, space probes and airplanes or photographs of our daily activists for different uses. Different techniques were invented for IP in the past 40-50 years, by using image of divergent source. IP acquired popularity due to the availability of personal computers, memory devices of wide capacity, graphics software, etc. It can be used in divergent application fields like remote sensing, medicine photographing, nondestructive evaluation, forensic experiment, textiles, military, cinema industries, document processing, graphic arts and print industry [2].

Known stages in the fields of IP are: - image scrutiny, storing, boosting and translation. This process is shown the diagram of image scanner-digitizer (see Figure 1).

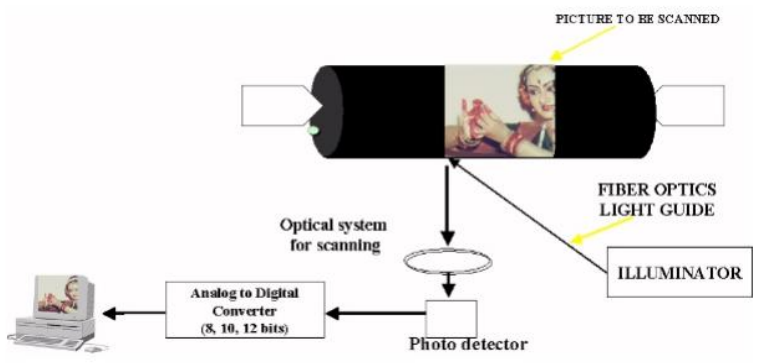

Fig1: Basic Image Processing Technique

[3] Suggested a defect feature extraction algorithm under HSV colour space depending upon the image processing. The suggested method able to discover defects from the background, kinds of defects of the underground pipelines could be classified in the estimation steps.

[4] introduced a substituted method to inspect defects presents in the pipes using novelty detection. A neural network was used to ignore normal conception, that suggested not any problem, therefore, objects that robot did not sense previously was highlighted 18 as a possible error. This decreases the possibility of false negatives. A novelty filter that can be operated on-line was also presented. Hence, every new input was evaluated for novelty regarding the data has been seen.

[5] Proposed an automatic and effective novel methodology for detection and identification of the defects in industrial pipelines, is based on image processing. This procedure has three steps. First, conversion the RGB image of the pipelines into a grayscale image. The second is extraction the pipelines and ultimately the detection and identification of the defects.

[6] Introduced a new methodology depending upon the laser generated ring onto the inner wall of the pipelines. Holes and defects location on the inner wall of the pipelines are detected via analysis the intensity of the projected rings of the light. Laser intensity is depending upon many factors, like the ring angle incidence or smoothness of the scattering surface. In ordinary values in one of these factors may result false defect detection. Therefore, it is necessary to hold enough knowledge of the laser reflectance phenomena for enhancement of the system performance and for realization its restrictions.

\section{MATERIALS TECHNIQUE}

\subsection{Feature Extraction Technique (FET)}

FE is one of the most important fields in artificial intelligence. It includes more suitable and interested features of an image and assign it into label. The substantial step in image categorization, is the analysis of the image qualities and 
arranges the numerical features into categories. It means, that the image is categorized on the basis of its contents [7, 8]. Categorization model and accuracy rate is depending upon the numerical qualities of different image features that represent the data of the categorization model. Recently, many (FET) have been developed each with its own strong and weak points. A suitable (FET) is the one that gives relevant features $[9,10]$.

\subsubsection{Edge detection (ED)}

In order to focus on the edge of an image, it is applicable that few weights of the filter be negative. A first-derivative row filter, at a location in the output image, produces variations in pixel values of the columns on both sides of this site of the input image. So, filters output would be grater (either negative or positive) when there are outstanding variations in the values of pixel in the right and left sides of the pixel site. A set of weights, which includes some smoothing in averaging over 3 rows is shown by $[11,12]$ :

$$
w=\frac{1}{6}\left(\begin{array}{lll}
-1 & 0 & 1 \\
-1 & 0 & 1 \\
-1 & 0 & 1
\end{array}\right)
$$

On the contrary second-derivative filters, may be similar and hence, respond to the edges in many orientations, if they remain linear. The arrangement is as follow:

$$
w=\frac{1}{3}\left(\begin{array}{rrr}
1 & 1 & 1 \\
1 & -8 & 1 \\
1 & 1 & 1
\end{array}\right)
$$

It is same kind of filter that is used to make (see Figure 2(c)), i.e. differences among the output from moving average filter as well as the raw image. With these weights, it can be shown that Laplacian transition of $f$ [12].

$$
\sum_{k=-1}^{1} \sum_{l=-1}^{1} w k l f_{i}+k, j+l \approx \frac{\partial^{2} f_{i j}}{\partial x^{2}}+\frac{\partial^{2} f_{i j}}{\partial y^{2}}
$$

Result of using of such filter to $x-$ ray image is gives (see Figure 2(a)). Edges manifest the image as zero-crossing in Laplacian that means the Laplacian will be +ve on one side and -ve in the other one. This is because an edge gives max. and min. values in the first derivative and zero value in the second derivative. (see Figure 2(b)) gives a tool to identify a zero-crossing, and the -ve and +ve values from the first filter shown as black and white respectively. Hence, zero-crossing match to black/white edges. For reducing the impact of the noise to the minimum level, the filter mentioned above or simple version of Laplacian filter is [12]:

$$
w=\left(\begin{array}{ccc}
0 & 1 & 0 \\
1 & -4 & 1 \\
0 & 1 & 0
\end{array}\right)
$$

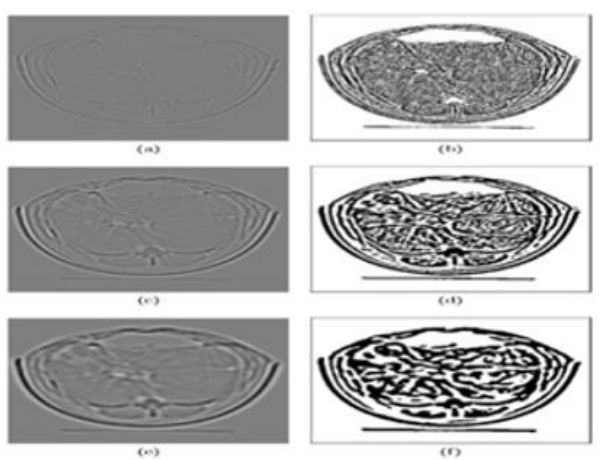

Fig 2: second-derivation filters applied to $x$-ray image (a) $3 * 3$ Laplacian filter, (b) zero-thresholded version of (a), (c) Laplacian-of-Gaussian filter, $\sigma^{2}=2$, (d) zero-thresholded version of (c), (e) Laplacian of Gaussian filter, $\sigma^{2}=2 / 3$,

(f) zero-thresholded version of (e)

There are three stages of edge detection, pre-processing, feature extraction FE and post-processing. ED process follow is shown (see Figure 3). After pre-processing, of image $I$, a middle result $I$ is resulted. This stage emphasizes on noise minimizing and matrix inhibition, while keeping edges as well as the absence of blurring edges between the divergent fields. The feature extraction has two stages: response computation and feature manipulation. In the first stage, estimation can come from statistical data, and a bunch of features $R$ is obtained [12].

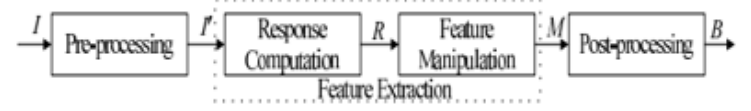

Fig 3: General edge detection flow

Feature manipulation stage includes, the choose and buildup of feature, so, the result is a bunch of features $M$. The FE stage is the main and necessary stage in edge detection. The aim of FE is to apply them to classify pixels as edge points or non-edge points. After post-processing, a final edge map $B$ is resulted. Post-processing mainly emphasizes many manipulating processes, marking edge points, thinning edges, removing stand-alone edge points and linking broken edge points. Generally, a normal pre-processing technique, such as Gaussian filtering, or a popular post-processing technique, such as thinning operations, can in general cooperate with various FET. This survey mainly covers the stage of extracting features. Most commonly used edge detection algorithms are Sobel, Canny, Prewitt, Roberts, zero-cross \& Laplacian of Gaussian [12].

\section{PROPOSE APPROACH}

Robots carrying digital cameras were inserted into non-metal sewer system underground pipelines. The cameras provided datasets as videos forms. These videos were processed by premade program converted them into frames. The results were one frame each one second. The frames were ultimately used the images containing additional aperture. Datasets were classified into classes used as inputs to detect the defects in the non-metal sewer underground pipelines using the following method. 


\subsection{Additional Apertures Detection}

Image with additional apertures (aperture of the joint between pipes) are characterized with discontinuity or sudden change in some optical properties such as, light intensity, structure or color. Therefore, for such image with these defects, edge detection technique was applied as shown down (see Figure 4).

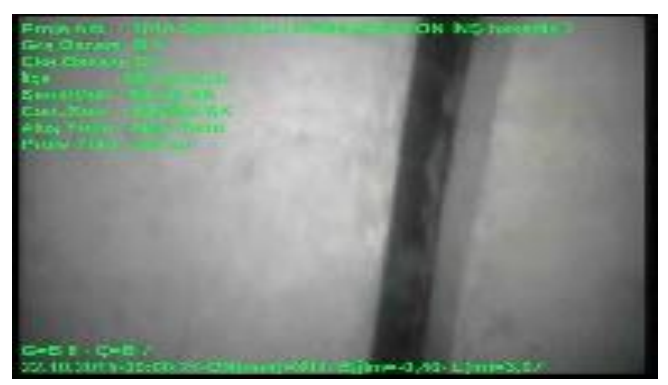

Fig 4: Additional aperture image

The basic concept of this technique is that, the edge information could be found through the relationship between each pixel in the image with the surrounding pixels. Edges in these types of image comprise specific useful information for image analysis and detect the additional apertures as well. Sobel or Prewitt operator with threshold equal to 0.020 was followed to detect the image edge. The function of this technique was to lineation of the objects of the image (i.e. additional apertures) by boosting the image brightness, eliminating many non-useful information in image analysis process. After applying the technique, bi-sector image with additional apertures boarders, was obtained, in addition to presence of some edges inside and outside the apertures boarders as noise as shown (see Figure 5).

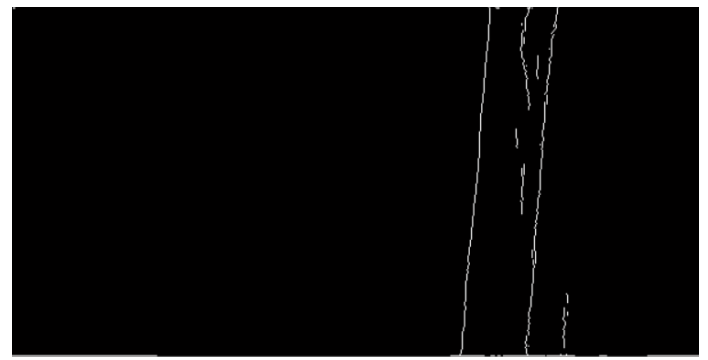

Fig 5: Sobel operator with threshold 0.020

The next step was the elimination of the noise inside and outside the object (apertures) by image close morphological technique, that is dilation followed by erosion. So, the result was logical image of background which is (0) value and additional apertures which have the value (1) for the pixels. (see Figure 6) bellow illustrate the output logical image.
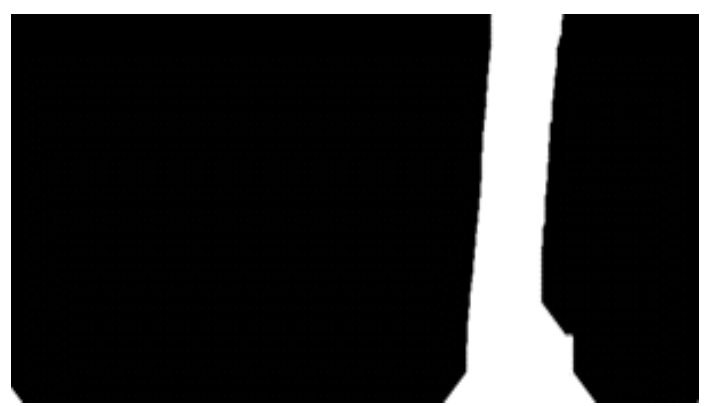

Fig 6: Logical Image
Finally, the properties of the object (apertures) were used as measurement unit for measurement and comparison to detect the image having additional apertures as defect.

This line was analyzed and its properties were extracted and used for distinguish the image containing such type of defects from the other images. For instance, the length of the object was measured and used to verify whether object is additional aperture or not. Length was also used for further analysis, if it is less than two-thirds of the image length so, this object is not additional aperture. On the other hand, object width has an importance not less than the importance of the length quality. Width has also been measured and used to identify the defect, in the sense that the width of the object does not exceed one third of the original image and not less than 20 pixels. Sometimes, the output may contain some other extra objects which are noises hindering the discovery process, whereas in this study this noises were exploited to be another feature help in the discovery process, in the sense that the image having defect, its noise does not exceed than (5) objects, otherwise, it is free of additional aperture.

\subsection{Accuracy of Proposed Algorithm}

To assess the additional aperture algorithm performance and accuracy for defect diagnosing, a group of edge detection operators were examined with median filter with various mask sizes. The proposed algorithm was applied for the available datasets using divergent groups of edge detection operators such as (Sobel, Prewitt, Canny and Roberts) with median filter with different mask sizes. Results in table (1) and (Figure 7) show that by using Sobel operator with mask sizes [5 5] and [7 7], Prewitt operator with mask sizes [3 3], [5 5], [7 7], [11 11] and [13 13], and Roberts operator with mask size [11 11] gave $100 \%$ accuracy regards the discovery of additional aperture, as compared with using Canny operator in different mask sizes where the results had been not satisfactory enough.

Table 1. Accuracy of proposed algorithm (\%)

\begin{tabular}{|c|c|c|c|c|c|c|}
\hline Edge operato & {$\left[\begin{array}{ll}3 & 3\end{array}\right]$} & {$\left[\begin{array}{ll}5 & 5\end{array}\right]$} & [7 7] & [9 9] & {$\left[\begin{array}{ll}11 & 1\end{array}\right]$} & [ $\left.\begin{array}{ll}13 & 13\end{array}\right]$ \\
\hline Sobel & 91.6 & 100 & 100 & 91.6 & 91.6 & 91.6 \\
\hline Canny & 33.6 & 33.6 & 33.6 & 33.6 & 33.6 & 33.6 \\
\hline Prewitt & 100 & 100 & 100 & 91.6 & 100 & 100 \\
\hline Roberts & 91.6 & 83.3 & 83.3 & 83.3 & 100 & 83.3 \\
\hline
\end{tabular}

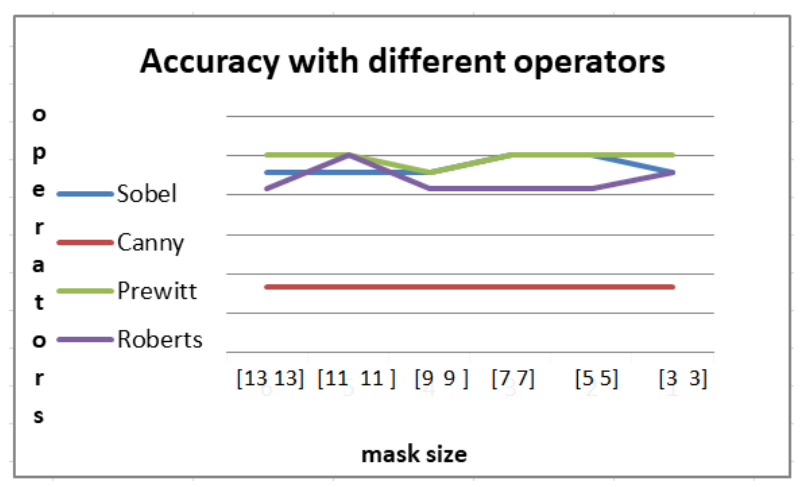

Fig7: Accuracy of the proposed algorithm with different edge detection operators 


\section{RESULTS AND DISCUSSION}

For simulation of the proposed work, MATLAB R2012a program was used in this study. This program's quality makes it efficient and suitable for easy and accurate applying such algorithms. By this program it was easy to analyze and represent giving data by processed it according to its datasets.

The accuracy and efficiency of the proposed algorithms were estimated through applying these algorithms on some datasets to obtain the accuracy desired via applying the following equations (2): -

$$
\begin{gathered}
\text { Precision }_{(i)}= \\
\frac{\text { correctly defects detected }_{(i)}}{\text { Total images tested containing } \operatorname{defect}_{(i)}}
\end{gathered}
$$

Where, $i=$ Additional aperture defect.

\subsection{The experiment (additional aperture)}

After the separation of the background from the part under study by applying the proposed algorithm to find out the additional aperture which is a line passing between regions of different properties (un-similar), accuracy of the proposed work was estimated, and shown in the table (2):

Table 2. Accuracy of Additional Aperture

\begin{tabular}{|c|c|}
\hline Algorithm & Accuracy \\
\hline Edge detection & 100 \\
\hline
\end{tabular}

\subsection{Discussion}

In this proposed work, edge detection technique was used to detect images having additional aperture. Result reflect high percent of accuracy $(100 \%)$, that may be attributed to the use of different mask sizes of median filter which improved the performance of the algorithm.

\section{CONCLUSION}

From the outstanding result of this study, it is clear that edge detection technique by estimating area (pixels numbers), length and width, swift clear and good detection for the additional aperture is resulted.

It is recommended beside using edge detection technique employing artificial intelligence techniques to detect anther types of defects in the sewer system.

\section{REFERENCES}

[1] Gonzalez, R. C. and Woods, R. E., 2008, Digital Image Processing $-3^{\text {rd }}$ ed., Upper Saddle River, New Jersey 07458, usa.
[2] Shefali, G. and kaur Y., 2014. Review of Different Contrast Enhancement Techniques for a Digital Image, I.J.A.R.C.SC.S. Eng. Vol.4 (7), pp 1213-1218.

[3] Wu, X. F. and Bai H., " Automated assessment of buried pipeline defects by image processing", Intelligent Computing and Intelligent Systems, 2009. ICIS 2009. IEEE International Conference.

[4] Stephen, M., Ulrich, N., and Jonathan, S., 2005. " Online novelty detection for autonomous mobile robots", 2005 Elsevier Robotics and Autonomous Systems 51 (2005), pp 191-206.

[5] Mayuri, D. S. and Prof. Kishor W., 2016. "An Application of Image Processing to Detect the Defects of Industrial Pipes", International Journal of Advanced Research in Computer and Communication Engineering Vol. 5, Issue 3, March 2016, pp 979-981.

[6] Safizadeh, M. S. and Azizzadeh, T., 2012. "Automated Detection of Inner Surface Defects in Pipes Using Image Processing Algorithms", Advanced Design and Manufacturing Technology, Vol. 5/ No. 5/ December 2012, pp53-59.

[7] Amin, S. S. and Ivanov, N., 2014. "Statistical Image Classification for Image Steganographic Techniques", International Journal of Image, Graphics and Signal Processing (IJIGSP), vol. 6, no. 8, pp. 19-24, 2014.DOI: 10.5815/ijigsp.2014.08.03.

[8] Morales, A. G., Acosta, N. M., Gago, A. A., García, E. B. R., and Medina, J. E. P., 2014. "A new proposal for graph-based image classification using frequent approximate subgraphs", Pattern Recognition, vol. 47, no. 1, pp. 169-177, 2014.

[9] Qian, J., Yang, J. and Gao, G., 2013. "Discriminative histograms of local dominant orientation (D-HLDO) for biometric image feature extraction”, Pattern Recognition, vol. 46, no. 10, pp. 2724-2739, 2013.

[10] Xiao, K., Liang, A. L., Guan, H. B. and Hassanien, A. E., 2013. "Extraction and application of deformation-based feature in medical images", Neurocomputing, vol. 120, pp. 177- 184, 2013.

[11] Moreno, R., Puig, D., Julia, C., and GARCIA, M., 2009. A new methodology for evaluation of edge detectors. In Proceedings of the 16th IEEE International Conference on Image Processing (2009), pp. 2157-2160.

[12] Wenlong, F., 2014. 'Feature Extraction in Edge Detection using Genetic Programming', PHD thesis, submitted to the Victoria University of Wellington. PP 17-19. 